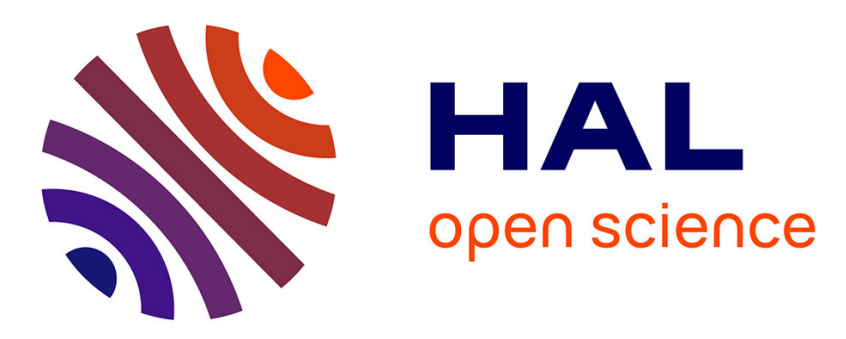

\title{
Numerical study of a macroscopic finite pulse model of the diffusion MRI signal
}

\author{
Jing-Rebecca Li, Hang Tuan Nguyen, Dang Van Nguyen, Houssem Haddar, \\ Julien Coatléven, Denis Le Bihan
}

\section{- To cite this version:}

Jing-Rebecca Li, Hang Tuan Nguyen, Dang Van Nguyen, Houssem Haddar, Julien Coatléven, et al.. Numerical study of a macroscopic finite pulse model of the diffusion MRI signal. Journal of Magnetic Resonance, 2014, pp.54-65. 10.1016/j.jmr.2014.09.004 · hal-01111058

\section{HAL Id: hal-01111058 \\ https://hal.science/hal-01111058}

Submitted on 29 Jan 2015

HAL is a multi-disciplinary open access archive for the deposit and dissemination of scientific research documents, whether they are published or not. The documents may come from teaching and research institutions in France or abroad, or from public or private research centers.
L'archive ouverte pluridisciplinaire HAL, est destinée au dépôt et à la diffusion de documents scientifiques de niveau recherche, publiés ou non, émanant des établissements d'enseignement et de recherche français ou étrangers, des laboratoires publics ou privés. 


\title{
Numerical study of a macroscopic finite pulse model of the diffusion MRI signal
}

\author{
Jing-Rebecca Li ${ }^{\mathrm{a}, \mathrm{b}}$, Hang Tuan Nguyen ${ }^{\mathrm{c}}$, Dang Van Nguyen ${ }^{\mathrm{b}}$, Houssem \\ Haddar $^{\mathrm{b}}$, Julien Coatléven ${ }^{\mathrm{b}}$, Denis Le Bihan ${ }^{\mathrm{c}}$ \\ ${ }^{a}$ Corresponding author, jingrebecca.li@inria.fr \\ ${ }^{b}$ INRIA Saclay-Equipe DEFI CMAP, Ecole Polytechnique, France \\ ${ }^{c}$ NeuroSpin, CEA Saclay Center 91191, Gif-sur-Yvette Cedex, FRANCE
}

\begin{abstract}
Diffusion magnetic resonance imaging (dMRI) is an imaging modality that probes the diffusion characteristics of a sample via the application of magnetic field gradient pulses. The dMRI signal from a heterogeneous sample includes the contribution of the water proton magnetization from all spatial positions in a voxel. If the voxel can be spatially divided into different Gaussian diffusion compartments with inter-compartment exchange governed by linear kinetics, then the dMRI signal can be approximated using the macroscopic Karger model, which is a system of coupled ordinary differential equations (ODEs), under the assumption that the duration of the diffusion-encoding gradient pulses is short compared to the diffusion time (the narrow pulse assumption). Recently, a new macroscopic model of the dMRI signal, without the narrow pulse restriction, was derived from the Bloch-Torrey partial differential equation (PDE) using periodic homogenization techniques. When restricted to narrow pulses, this new homogenized model has the same form as the Karger model.
\end{abstract}

We conduct a numerical study of the new homogenized model for voxels that are made up of periodic copies of a representative volume that contains spherical and cylindrical cells of various sizes and orientations and show that the signal predicted by the new model approaches the reference signal obtained by solving the full Bloch-Torrey PDE in $O\left(\varepsilon^{2}\right)$, where $\varepsilon$ is the ratio between the size of the representative volume and a measure of the diffusion length. When the narrow gradient pulse assumption is not satisfied, the new homog- 
enized model offers a much better approximation of the full PDE signal than the Karger model. Finally, preliminary results of applying the new model to a voxel that is not made up of periodic copies of a representative volume are shown and discussed.

Keywords: Diffusion MRI; signal model; homogenization; effective medium; macroscopic model; Karger model.

\section{Introduction}

The image contrast in water proton diffusion magnetic resonance imaging (dMRI) comes from the differing water diffusion characteristics in the imaged tissue at different spatial positions [1]. A major application has been in acute cerebral ischemia (stroke) $[2,3]$. DMRI has been used to detect and differentiate a wide range of physiological and pathological conditions, including, in the brain, tumors $[4,5,6]$, myelination abnormalities (for a review, see [7]), as well as in the study of brain connectivity (for a review, see [8]) and in functional imaging [9].

The signal measured by the MRI scanner is a mean-value measurement in a physical volume, called a voxel, whose size is much larger than the scale of the microscopic variations of the cellular structure. The resolution of dMRI is on the order of $1 \mathrm{~mm}^{3}$, meaning the dMRI signal averages the diffusion characteristics of a tissue volume (voxel) of $1 \mathrm{~mm}^{3}$. This is very large compared to cell features, which vary from sub- $\mu$ m (diameter of neurites) to tens of $\mu \mathrm{m}$ (diameter of neuronal bodies and glial cells) in the brain. In other words, dMRI is used to show the averaged characteristics of the microscopic structure on a macroscopic scale. Another very important spatial scale to consider is the diffusion displacement of water molecules during the measured diffusion time. At physically realistic dMRI diffusion times of 10-100 ms, the average diffusion displacement is, assuming a mean diffusivity of $10^{-3} \mathrm{~mm}^{2} / \mathrm{s}$, between 8-25 $\mu \mathrm{m}$. Thus, from the point of view of diffusing water molecules, their diffusion displacement is large with respect to some cell features (diameter of neurites) but not with respect to others (diameter of neuronal bodies and glial cells). In addition, due to the presence of cell membranes, which hinder the movement of water, the diffusion displacements of water molecules may be very strongly dependent on their positions (whether in the neurites, in the neuronal bodies or glial cells, or in the extra-cellular space) 
within the voxel. An additional difficulty in understanding the dMRI signal is that even though the cell membranes hinder the movement of water across them, there may still be significant water exchange across the membranes that contributes to the dMRI signal. In fact, assigning a diffusional permeability coefficient to the cell membranes is challenging (see [10] and references therein).

There have been many proposed macroscopic models of the dMRI signal in tissue, ranging from simple to complicated, each valid under a certain set of assumptions.

A simple model is the Gaussian model, where the entire voxel is described as a macroscopically Gaussian diffusion environment, and the dMRI signal is described by a decaying exponential, associated to an effective diffusion tensor. If the voxel is assumed to be made up of periodic copies of a representative volume, the effective diffusion tensor for general cell shapes can be obtained by solving a set of Laplace PDEs [11]. For simple geometries such as cubes and spheres, analytical formulae for the effective diffusion tensor can be found in $[12,13,14,15]$. A recent review of theoretical models for the dMRI signal can be found in [16], including references to many additional works on Gaussian models that we do not mention here.

Unfortunately, there is ample experimental evidence that the diffusion in brain tissue at diffusion times relevant to dMRI is not macroscopically Gaussian: the dMRI signal has been fitted as multiple compartmental Gaussian [17, 18, 19, 20], or by including a Kurtosis term to quantify the nonGaussianness [21, 22], or by fitting with fractional order diffusion [23].

To generalize beyond macroscopically Gaussian models, we mention the statistical model of [24], where the spin packets are divided into groups and each group is assumed to undergo macroscopic Gaussian diffusion with a different effective diffusion tensor. These groups are not explicitly defined but are assumed to be distributed according to some peaked distribution function. There is also the work of [25] where the expansion of the dMRI signal around a perturbation of the mean diffusivity is considered. In [26] the time dependence of the average diffusion distance in the presence of compact cells and permeable membranes is considered. These models have the advantages of being rather general descriptions of the dMRI signal, however, they do not provide explicit links between the dMRI signal and biological quantities such as the volume fraction of neurites, neuronal bodies and glial cells, and 
the cell membrane permeability. At least, making more explicit connections between these models and the above biological quantities would require further analysis. We also mention a recent work [27] that studies diffusion in both ordered as well as different types of disordered systems that has the membrane permeability and surface to volume as parameters.

Next, there are models that explicitly define tissue compartments. For example, the models of $[28,29,30]$ separate the cylindrical-shaped neurites from the space outside them to make two diffusion compartments and assume there is no water exchange between them. When there is no water exchange between the diffusion compartments, the total dMRI signal is just the sum of the signals from each of the compartments. We note that in these noexchange models, one can in fact easily allow non-Gaussian diffusion in each compartment, and this flexibility may potentially result in the applicability of the models at shorter diffusion times.

Since it is not clear whether the non-Gaussian nature of the dMRI signal is due to the non-Gaussian nature of the diffusion in the different tissue compartments, or the water exchange between the compartments, or both, it is also useful to examine signal models that take into account intercompartmental exchange. A multiple compartment macroscopic model for dMRI that allows exchange between the compartments is the Karger model [31], which was originally developed for micro-porous crystallites. The Karger model is obtained by supposing multiple Gaussian diffusion compartments, each of which covers $\mathbb{R}^{d}$, where $d$ is the space dimension, and where the spin exchange between the compartments is described by simple constant rate terms that can be added to the diffusion terms. These assumptions enable the formulation of a system of coupled ODEs that describes the time evolution of the signals in the different compartments. The dMRI signal of the Karger model is the sum of all the components of the unknowns of the ODE system at the diffusion time and the ODE system, which has constant coefficients, is easy to solve.

The Karger model was obtained on the basis of phenomenological modeling of the experimentally obtained signal curves and is valid under the narrow pulse assumption: meaning the duration of the gradient pulses should be short compared to the diffusion time.

Recently, a new macroscopic model for the dMRI signal was formulated in [32] using periodic homogenization techniques on the Bloch-Torrey PDE in 
heterogeneous domains. Periodic homogenization supposes that the macroscopic domain $\Omega$, of the spatial scale $L$, is made up of periodic copies of a representative volume $C$ (for simplicity, a cube), of side length $l$. Starting from a PDE defined on $\Omega$, using homogenization techniques, a related, homogenized, PDE can be formulated whose solution is close to the solution of the original PDE. In [32] the resulting homogenized PDE was solved to obtain a coupled ODE system that describes the time evolution of the magnetization in each tissue compartment. The compartments are defined from the outset as the biological cells and the extra-cellular space. There is no ambiguity as to the meaning of the different compartments. Like the Karger model, the dMRI signal is obtained after solving a system of ODEs, but the ODEs have time-dependent coefficients and not constant coefficients like the Karger model. The main advantage of this new macroscopic model is that it is not subject to the narrow pulse restriction. The gradient pulses can be long in duration and can have an arbitrary shape. In the following, we will call this new macroscopic model the Finite Pulse Karger (FPK) model for the dMRI signal. In the narrow pulse limit, the FPK model has the same form as the Karger model.

Because of the focus of [32] is on the mathematical derivation of the FPK model, only very simple numerical simulations (in two dimensions, with circular cells) were performed. In the current paper, we conduct a more thorough numerical study of the FPK model. First, we will assume that the voxel is made up of periodic copies of a representative volume $C$. This is the assumption underlying periodic homogenization. We construct representative volumes $C$ containing spheres and long cylinders of various sizes and orientations so as to have a variety of diffusion characteristics in the different compartments. As a reference solution, we solve the Bloch-Torrey equation on the heterogeneous domains. We show numerically that the FPK signal approaches the reference signal in $O\left(\varepsilon^{2}\right)$, where $\varepsilon$ is the homogenization parameter that we defined as the ratio between the size of the representative volume and a measure of the diffusion length. We also show when the narrow pulse assumption is not satisfied, the FPK model offers a much better approximation of the reference signal than the Karger model. Finally, we show and discuss preliminary results of applying the FPK model to a voxel that is not made up of periodic copies of a representative volume.

The main practical interest in having a valid macroscopic model for the dMRI signal is that the parameter estimation problem of determining model 
parameters from the experimental signal would be relatively easily solvable and it is hoped that the macroscopic model parameters can give useful insights into the underlying tissue structure. For example, the macroscopic model parameters of the FPK model are the effective diffusivities and the volume fractions of the different diffusion compartments, as well as the intercompartmental exchange times. Ideally, the diffusion compartments would be able to be identified to physical tissue compartments such as a compartment comprised of neurites, a compartment comprised of cells of non-elongated shapes, a compartment comprised of the extra-cellular space. In this case, the effective diffusivities could be potentially related to geometrical properties of the compartments, such as cell shape and arrangement. Similarly, it is hoped that the exchange times could be related to membrane permeability and the surface to volume ratio of the compartments. In summary, once a valid macroscopic model has been found, two steps to make use of it are 1 . estimating the macroscopic model parameters that best fit the experimental signal, subject to noise (for example, by solving a least squares problem); 2. interpreting the macroscopic model parameters in terms of tissue properties (which is itself a difficult problem requiring further analysis or numerical simulations).

Whether the FPK model is or can be generalized to a valid macroscopic model for brain tissue dMRI is a difficult question to answer at this point. The fact that the FPK model was derived in [32] using periodic homogenization does not necessarily limit its use to periodic domains. A simple identification of the macroscopic model parameters of the FPK model to that of the Karger model can be done, and in this way we obtain a generation of the Karger model to non-narrow pulse sequences. At the very least, if the Karger model is not limited to periodic domains, then the FPK is not either, once the reinterpretation or re-definition of the macroscopic model coefficients is done. We cite the non-periodic homogenization approach in porous media [33, 34], where the difference between the periodic and the non-periodic cases is in the definition and interpretation of the macroscopic model coefficients. Further study is needed to evaluate the PFK model beyond periodic domains to test its validity in realistic geometries of brain tissue.

The more relevant issue is that even though the Karger model has already been used in biological tissue dMRI to invert for model parameters in [35, $36,37,38,10,39,40,41,42]$, the validity of the Karger model to biological tissue, which is certainly not a periodic medium, would benefit from further 
investigation. In [43] it was stated that for the Karger model to be applicable, there are three conditions:

- The diffusion is Gaussian in each compartment separately;

- The diffusion length exceeds the cell packing correlation length;

- The exchange between the compartments is slow compared to the characteristic time to traverse the correlation length of the cell packing.

These statements are quite difficult to interpret and verify, for example, even when considering a reasonable geometrical model of brain tissue such as a voxel-sized domain containing densely packed dendrite trees and cylindrical axons with some orientation distribution and spherical cells modeling soma and glia cells. Hence we consider numerical simulations on voxel-sized (three dimensional) domains that contain densely packed, randomly placed and oriented cells of spherical and cylindrical geometries (and tree structures) to verify the above statemetns an extremely useful future direction. We illustrate this by showing preliminary results of applying the FPK model to a two dimensional voxel-sized non-periodic domain in Section 5. In this paper, we study the FPK model for periodic domains because the macroscopic model parameters can be defined rigorously for periodic domains, and we are able to show the convergence of the FPK model to the microscopic model in the homogenization parameter $\varepsilon$ in a clear way. We hope that these results can then guide us improving and generalizing macroscopic models to brain tissue dMRI.

\section{Theory}

\subsection{Tissue model}

We define a simplified geometrical model of brain tissue that consists of spheres and/or long cylinders of various orientations embedded in the extracellular space. The spheres will represent the neuronal bodies and the glial cells, and the cylinders will represent the neurites. We will assume that the water exchange between the neuronal body and the neurites attached to it to be small enough to be negligible, because the intersection of the neuronal body with the attached neurites has a small surface area. 
Let $V$ be the voxel of interest. We assume that $V$ is made up of periodic copies of a representative volume that is, for simplicity, a cube $C=\left[-\frac{l}{2}, \frac{l}{2}\right]^{3}$, such that

$$
V=\bigcup_{m, n, j \in\{\cdots,-1,0,1, \cdots\}} C+(m l, n l, j l) .
$$

We will not be concerned with the effect of the neighboring voxels on $V$ because the diffusion distance in brain dMRI, around tens of $\mu \mathrm{m}$, is much smaller than the side lengths of the voxel (on the order of $1 \mathrm{~mm}$ ). This justifies the simplification that we will border $V$ by identical copies of itself instead of its true neighbors. Thus, from the point of view of water molecules starting in $C$, the spatial domain is made up of copies of $C$ during the diffusion-encoding sequence. Obviously, if we want to place long cylindrical cells in the voxel, we must construct the cylinders so that they are oriented in such a way that when $C$ is periodically repeated, the cylinders are not "broken" at the faces of $C$.

\subsection{Reference dMRI signal computed from Bloch-Torrey PDE}

The reference (microscopic) model against which we will compare the FPK and Karger macroscopic models is the multiple compartment Bloch-Torrey partial differential equation (PDE) $[44,45]$. In the following we do not include the dependence of the MRI signal on the imaging gradients and the $T_{2}$ relaxation so we can simplify the presentation. For the same reason, we also make the intrinsic diffusion coefficient the same in all the compartments, denoting it by $D^{0}$, the permeability the same on all the membranes, denoting it by $\kappa$, and the spin density the same in all compartments.

The representative volume $C$ will be our computational domain. Inside $C$, there will be an extra-cellular compartment $\Omega^{e}$. For simplicity, we assume $\Omega^{e}$ is connected (otherwise, it needs to be broken into connected components). In $C$ there also will be sphere compartments, $\Omega^{s_{j}}, j=1, \cdots$, where each $\Omega^{s_{j}}$ is a sphere, and cylinder compartments, $\Omega^{c_{k}}, k=1, \cdots$, where each $\Omega^{c_{k}}$ is a (part of a long) cylinder. The union of the compartments is $C=\bigcup_{p=\left\{e,\left\{s_{j}\right\},\left\{c_{k}\right\}\right\}} \Omega^{p}$. Since $C$ is periodically repeated, obviously, so are the $\Omega^{p}$ 's. Finally, we note that one could also have membrane compartments, consisting of a thick layer of space around the surfaces of the cylinders and the spheres.

For the macroscopic models to be discussed later, we can also group all cells with similar diffusion characteristics into a single compartment to simplify 
things. For example, if we do not want to distinguish between spheres of different radii, then we can define the union of all the spheres as one compartment in the macroscopic model. This redefinition makes no difference for the microscopic PDE model since each sphere is always distinct from the others but it is notationally convenient when we want to compare the PDE model and the macroscopic models compartment by compartment.

The complex transverse water proton magnetization $M^{p}(\mathbf{x}, t \mid \mathbf{g})$ in each compartment $\Omega^{p}$ satisfies the Bloch-Torrey PDE [44]:

$$
\frac{\partial M^{p}(\mathbf{x}, t \mid \mathbf{g})}{\partial t}=-I f(t)(\gamma \mathbf{g} \cdot \mathbf{x}) M^{p}(\mathbf{x}, t \mid \mathbf{g})+\nabla \cdot\left(D^{0} \nabla M^{p}(\mathbf{x}, t \mid \mathbf{g})\right), \quad \forall p,
$$

where we denote the amplitude and direction of the diffusion-encoding gradient by $\mathbf{g}=\left(g_{1}, g_{2}, g_{3}\right)$ and its time profile by $f(t), I$ is the imaginary unit, $\gamma=42.576 \mathrm{MHz} /$ Tesla is the gyromagnetic ratio of the water proton.

For the pulsed gradient spin echo (PGSE) sequence[46], with two rectangular pulses of duration $\delta$, separated by a time interval $\Delta-\delta$, the profile $f(t)$ is

$$
f(t)= \begin{cases}1, & t_{1} \leq t \leq t_{1}+\delta \\ -1, & t_{1}+\Delta<t \leq t_{1}+\Delta+\delta \\ 0, & \text { otherwise }\end{cases}
$$

where $t_{1}$ is the starting time of the first gradient pulse, with $t_{1}+\Delta>T E / 2$, the $T E$ is the echo time.

We supplement the PDE in (1) with interface conditions where $\Omega^{p}$ and $\Omega^{n}$ come in contact. We denote the interface between $\Omega^{p}$ and $\Omega^{n}$ by $\Gamma^{p n}$. One interface condition is the continuity of flux:

$$
D^{0}\left(\nabla M^{p}(\mathbf{y}, t \mid \mathbf{g}) \cdot \mathbf{n}^{p}(\mathbf{y})\right)=-D^{0}\left(\nabla M^{n}(\mathbf{y}, t \mid \mathbf{g}) \cdot \mathbf{n}^{n}(\mathbf{y})\right), \mathbf{y} \in \Gamma^{p n},
$$

where $\mathbf{n}^{p}(\mathbf{y})$ and $\mathbf{n}^{n}(\mathbf{y})$ are the outward-point normals to $\Omega^{p}$ and $\Omega^{n}$ at $\mathbf{y}$, so in fact $\mathbf{n}^{p}(\mathbf{y})=-\mathbf{n}^{n}(\mathbf{y})$. This ensures the conservation of the magnetization. The second interface condition is:

$$
D^{0}\left(\nabla M^{p}(\mathbf{y}, t \mid \mathbf{g}) \cdot \mathbf{n}^{p}(\mathbf{y})\right)=\kappa\left(M^{p}(\mathbf{y}, t \mid \mathbf{g})-M^{n}(\mathbf{y}, t \mid \mathbf{g})\right), \mathbf{y} \in \Gamma^{p n} .
$$

This incorporates a permeability coefficient $\kappa$ across $\Gamma^{p n}$ and models the ease with which water crosses the interface. The larger the $\kappa$, the easier the 
passage of water. Now we add the initial condition:

$$
M(\mathbf{x}, 0 \mid \mathbf{g})=\rho, \quad \mathbf{x} \in \Omega^{p}, \quad \forall p,
$$

where we assumed the same spin density $\rho$ in all the $\Omega^{p}$ 's. Then, same as [47], to mimic the effect of a diffusion domain that contains periodic copies of $C=\left[-\frac{l}{2}, \frac{l}{2}\right]^{3}$, the boundary conditions to impose on $\partial C$ are:

$$
\begin{gathered}
\left.M(\mathbf{x}, t \mid \mathbf{g})\right|_{x_{k}=-\frac{l}{2}}=\left.M(\mathbf{x}, t \mid \mathbf{g})\right|_{x_{k}=\frac{l}{2}} e^{I \theta_{k}(t)}, \quad k=1,2,3, \\
\left.\frac{\partial M(\mathbf{x}, t \mid \mathbf{g})}{\partial x_{k}}\right|_{x_{k}=-\frac{l}{2}}=\left.\frac{\partial M(\mathbf{x}, t \mid \mathbf{g})}{\partial x_{k}}\right|_{x_{k}=\frac{l}{2}} e^{I \theta_{k}(t)}, \quad k=1,2,3,
\end{gathered}
$$

for each of the faces perpendicular to the three coordinate axes, where $\mathbf{x}=$ $\left(x_{1}, x_{2}, x_{3}\right)$ and $\mathbf{g}=\left(g_{1}, g_{2}, g_{3}\right)$, and

$$
\theta_{k}(t)=\gamma g_{k} l \int_{0}^{t} f(s) d s
$$

Thus, the complete mathematical problem of the microscopic multiple compartment Bloch-Torrey PDE for a representative volume $C$ consists of the PDE (Eq. 1), the interface conditions (Eq. 3, Eq. 4), the initial condition (Eq. 5), and the boundary conditions (Eq. 6, Eq. 7).

Now we will make the very important definition of the compartment magnetizations of the PDE model as the integral of the magnetization in $\Omega^{p}$ :

$$
\bar{M}_{P D E}^{p}(b, t):=\int_{\mathbf{x} \in \Omega^{p}} M^{p}(\mathbf{x}, t \mid \mathbf{g}) d \mathbf{x}, \quad 0 \leq t \leq T E,
$$

where, following convention, the independent variable $b$, the b-value, is defined as:

$$
b(\mathbf{g})=\gamma^{2}\|\mathbf{g}\|^{2} \int_{0}^{T E} d u\left(\int_{0}^{u} f(s) d s\right)^{2} .
$$

We emphasize that the compartment magnetizations depend on $t$ and the compartment index $p$. The dMRI signal measured in experiments (without the imaging gradients and $T_{2}$ effects) corresponds to

$$
S_{P D E}(b):=\sum_{p} \bar{M}_{P D E}^{p}(b, T E)=\sum_{p} \int_{\mathbf{x} \in \Omega^{p}} M^{p}(\mathbf{x}, T E \mid \mathbf{g}) d \mathbf{x} .
$$


Because $C$ is assumed to be periodically repeated in the voxel $V$, the above integrals of the magnetization can be taken over $C$ only. The signal is the same as if the magnetization is taken over $V$ (and correctly normalized).

We note that even though the total magnetization (and hence the dMRI signal $\left.S_{P D E}(b)\right)$ can be measured only at the echo time and combines the contributions from all the compartments, the compartment magnetizations $\bar{M}_{P D E}^{p}(b, t)$ defined in Eq. 8, are meaningful mathematically for all $t \leq T E$ and all $p$. By computing the compartment magnetizations we gain insight about the time evolution of the magnetizations in the different compartments and how they mix.

In a dMRI experiment, the $T E$ and sequence $f(t)$ are usually fixed while $\mathbf{g}$ is varied in amplitude and in direction to obtain the signal at different b-values. From the acquired signal at some b-values, a quantity called the "Apparent Diffusion Coefficient" can be computed for each voxel. This quantity correponds in our notation to

$$
A D C_{0}:=-\left.\frac{\partial}{\partial b} \log \frac{S_{P D E}(b)}{S_{P D E}(0)}\right|_{b=0},
$$

where we denoted the quantity by $A D C_{0}$ to emphasize that the analytical derivative of $\log S(b)$ is taken at $b=0$. In the narrow pulse limit, the $A D C_{0}$ gives the mean squared distance traveled by water molecules, averaged over all starting positions.

In the following, we will set the spin density to $\rho=\frac{1}{|C|}$, so that $S_{P D E}(b=0)=$ 1 and $\bar{M}_{P D E}^{p}(b, t=0)=\frac{\left|\Omega^{p}\right|}{|C|}:=v^{p}$, where $\left|\Omega^{p}\right|$ is the volume of $\Omega^{p},|C|$ is the volume of $C$, and $v^{p}$ is the volume fraction of $\Omega^{p}$. Then the signal $S_{P D E}(b)$ will be automatically normalized and it is also the signal attenuation.

\subsection{Karger model}

In the formulation of the Karger model [48], one starts with a system of PDEs for the concentration, $u^{p}(\mathbf{x}, t)$, where $\mathbf{x}$ is the macroscopic spatial variable, of water in $W$ Gaussian diffusion compartments: $p=1, \cdots, W$. The governing equations for the diffusion within and the exchange between 
the compartments are:

$$
\begin{aligned}
\frac{\partial u^{1}(\mathbf{x}, t)}{\partial t} & =\nabla \bar{D}_{e f f}^{1} \nabla u^{1}(\mathbf{x}, t)-\frac{u^{1}(\mathbf{x}, t)}{\omega^{1}}+\sum_{p=2, \cdots, W} \frac{u^{p}(\mathbf{x}, t)}{\tau^{1 p}}, \\
& \vdots \\
\frac{\partial u^{W}(\mathbf{x}, t)}{\partial t} & =\nabla \bar{D}_{e f f}^{W} \nabla u^{W}(\mathbf{x}, t)-\frac{u^{W}(\mathbf{x}, t)}{\omega^{W}}+\sum_{p=1, \cdots, W-1} \frac{u^{p}(\mathbf{x}, t)}{\tau^{W p}},
\end{aligned}
$$

where $\mathbf{x} \in \mathbb{R}^{3}, \tau^{p n}$ is the exchange time between the compartment $p$ and $n$, and $\bar{D}_{\text {eff }}^{p}$, when using the Karger model in biological tissue dMRI applications, is the effective diffusion tensor of compartment $p$. We note here that the best way to define the effective diffusion tensors $\bar{D}_{\text {eff }}^{p}, p=1 \cdots W$, for realistic geometries of brain tissue is in fact a very difficult problem that requires further investigation (see a preliminary discussion in Sec 5). From the mass conservation, if we assume that the spin density is the same in all the compartments, then $\omega^{1}, \cdots, \omega^{W}$, must satisfy:

$$
\frac{1}{\omega^{n}}=\frac{1}{v^{n}} \sum_{p=1, p \neq n}^{W} \frac{v^{p}}{\tau^{n p}}, \quad n=1, \cdots, W,
$$

where $v^{p}$ is the volume fraction of compartment $p$. The system of PDEs in (12) are subject to initial conditions:

$$
u^{p}(\mathbf{x}, 0)=v^{p} \tilde{\delta}(\mathbf{x}), p=1, \cdots, W
$$

where $\tilde{\delta}(\mathbf{x})$ is the Dirac delta distribution at $\mathbf{x}=0$. We used the notation $\tilde{\delta}$ to distinguish it from the width of the gradient pulse $\delta$ in the PGSE sequence.

In the Karger model, the compartment magnetization $\bar{M}_{K A R}^{p}(b, t)$, arising from compartment $p$, under the narrow pulse approximation for the PGSE sequence, $\delta \ll \Delta$, has the following form:

$$
\bar{M}_{K A R}^{p}(b, t)=\int_{\mathbf{x} \in \mathbb{R}^{3}} e^{I \gamma \delta \mathbf{g} \cdot \mathbf{x}} u^{p}(\mathbf{x}, t) d \mathbf{x} .
$$

We note that the region of integration in Eq. 15 is $\mathbb{R}^{3}$. Taking the time derivative of $\bar{M}_{K A R}^{p}$ in Eq. 15 and using Green's identity, the Karger model 
can be obtained:

$$
\begin{aligned}
\frac{d \bar{M}_{K A R}^{n}(b, t)}{d t}= & -\left(\delta^{2} \gamma^{2} \mathbf{g}^{T} \bar{D}_{e f f}^{n} \mathbf{g}+\frac{1}{v^{n}} \sum_{p=1, p \neq n}^{W} \frac{v^{p}}{\tau^{n p}}\right) \bar{M}_{K A R}^{n}(b, t) \\
& +\sum_{p=1, p \neq n}^{W} \frac{1}{\tau^{n p}} \bar{M}_{K A R}^{p}(b, t), \quad n=1, \cdots, W .
\end{aligned}
$$

It is a system of coupled ODEs, subject to the initial conditions:

$$
\bar{M}_{K A R}^{p}(b, 0)=v^{p}, p=1, \cdots, W .
$$

The analytical solution of (16) can be obtained if the $W \times W$ matrix on the right hand side of Eq. 16 has an eigen-decomposition.

In the original Karger model, the dMRI signal is the sum of the $\bar{M}_{K A R}^{p}$ 's from all the compartments at $t=\Delta$ :

$$
\sum_{p=1}^{W} \bar{M}_{K A R}^{p}(b, \Delta) \text {. }
$$

To best compensate for a finite width $\delta$, we evaluate the Karger model at $t=\Delta-\delta / 3$ to obtain

$$
S_{K A R}(b)=\sum_{p=1}^{W} \bar{M}_{K A R}^{p}(b, \Delta-\delta / 3),
$$

for the Karger signal when comparing the Karger model with the FPK model to be described next. The reasoning is similar to that for a homogeneous system with diffusion tensor $\bar{D}$, where the dMRI signal is $e^{\gamma^{2} \delta^{2} \mathbf{g}^{T} \bar{D} \mathbf{g}(\Delta-\delta / 3)}$, which "looks like" a "diffusion time" of $(\Delta-\delta / 3)$ is being evaluated to compensate for the finite pulse. The form of the Karger model suggests the evaluation at $t=\Delta-\delta / 3$ because the ODE system in Eq. 16 imposes the instantaneous phase accumulation $\gamma \delta \mathbf{g}$, as can be seen by the term $\delta^{2} \gamma^{2} \mathbf{g}^{T} \bar{D}_{\text {eff }}^{n} \mathbf{g}$. To have the Karger model in Eq. 16 give the correct signal for the homogeneous case, the evaluation time point of the ODE system should be $t=\Delta-\delta / 3$ (in contrast to $t=\Delta+\delta$, for example). The $A D C_{0}$ of the Karger model is

$$
A D C_{0}^{K A R}=\sum_{p=1}^{W} v^{p} \frac{\mathbf{g}^{T} \bar{D}_{e f f}^{p} \mathbf{g}}{\|\mathbf{g}\|^{2}} .
$$




\subsection{Finite Pulse Karger model}

The derivation of the finite pulse Karger model[32] starts with the multiple compartment Bloch-Torrey PDE (Eqs. 1-7) and uses periodic homogenization theory [49], where the voxel was assumed to be a periodic repetition of a representative volume $C$ (see Appendix for details). The final form of the

model obtained in [32] is a set of ODEs governing the time evolution of the compartment magnetizations defined in Eq. 8:

$$
\begin{aligned}
\frac{d \bar{M}_{F P K}^{m}(b, t)}{d t}= & -\left(c(t) \gamma^{2} \mathbf{g}^{T} \bar{D}_{e f f}^{m} \mathbf{g}+\frac{1}{v^{m}} \sum_{p=1, p \neq m}^{W} \frac{v^{p}}{\tau_{F P K}^{m l}}\right) \bar{M}_{F P K}^{m}(b, t) \\
& +\sum_{p=1, p \neq m}^{W} \frac{1}{\tau_{F P K}^{m l}} \bar{M}_{F P K}^{p}(b, t), \quad m=1, \cdots, W,
\end{aligned}
$$

where the time-dependent coefficient function $c(t)$ is:

$$
c(t) \equiv\left(\int_{0}^{t} f(s) d s\right)^{2},
$$

and if each $\Omega^{p} \in C$ is connnected, then

$$
\frac{1}{\tau_{F P K}^{m l}}:=\kappa \frac{\left|\Gamma^{m l}\right|}{\left|\Omega^{p}\right|}, \quad l \neq m,
$$

where $\left|\Gamma^{m l}\right|$ is the surface area between $\Omega^{m}$ and $\Omega^{p}$, and $\left|\Omega^{p}\right|$ is the volume of $\Omega^{p}$. The effective diffusion tensors under the assumption of periodic homogenization are shown to be steady-state values

$$
\bar{D}_{e f f}^{m}=\bar{D}_{s s}^{m},
$$

defined as:

$$
\left(\bar{D}_{s s}^{m}\right)_{j, k} \equiv \frac{1}{v^{m}} \int_{\Omega^{m}} D^{0} \nabla M_{j}^{s s}(\mathbf{x}) \cdot \mathbf{e}_{k} d \mathbf{x}, \quad j, k=1, \cdots, 3,
$$

where $\mathbf{e}_{k}$ is the unit vector in the $k^{\text {th }}$ direction, after solving three Laplace (state-state) PDEs in $C$ for the steady-state functions $M_{1}^{s s}, M_{2}^{s s}, M_{3}^{s s}$ :

$$
\nabla \cdot\left(D^{0} \nabla M_{j}^{s s}(\mathbf{x})\right)=0, \quad \mathbf{x} \in \Omega^{m}, \quad j=1,2,3,
$$


subject to impermeable boundary condition on $\partial \Omega^{m}$ :

$$
\nabla M_{j}^{s s}(\mathbf{y}) \cdot \mathbf{n}(\mathbf{y})=0, \quad \mathbf{y} \in \partial \Omega^{m}, \quad j=1,2,3,
$$

and boundary conditions on $\partial C$ :

$$
\begin{aligned}
\left.M_{j}^{s s}(\mathbf{x})\right|_{x_{k}=-l / 2} & =\left.M_{j}^{s s}(\mathbf{x})\right|_{x_{k}=l / 2}-w_{j, k} L_{k}, k=1, \cdots, 3, \\
\left.\frac{\partial}{\partial x_{k}} M_{j}^{s s}(\mathbf{x})\right|_{x_{k}=-l / 2} & =\left.\frac{\partial}{\partial x_{k}} M_{j}^{s s}(\mathbf{x})\right|_{x_{k}=l / 2}, \quad k=1, \cdots, 3,
\end{aligned}
$$

where $w_{j, k}=1$ if $k=j$, and $w_{j, k}=0$, otherwise.

There are three important things to note:

1. The macroscopic parameter $\bar{D}_{s s}^{m}$ is the effective diffusivity in $\Omega^{m}$ in the infinite time limit and it can be un-ambiguously defined for a periodic domain. One may call $\bar{D}_{s s}^{m}$ the "tortuosity limit" if $\Omega^{m}$ is an unrestricted domain.

2. The permeability coefficient does not enter into the definition of $\bar{D}_{s s}^{m}$.

3. $\bar{D}_{s s}^{m}$ is a physical quantity of the cellular geometry, it does not depend on the diffusion-encoding gradient.

For a sphere compartment, it is not necessary to actually solve Eqs. 25-28. It is easy to show that

$$
\text { (sphere) } \bar{D}_{s s}^{m}=0 \text {. }
$$

We note that the above is true for any geometry of finite size (in all directions). For the cylinder compartments, we will construct the computational domain $C$ so that when it is periodically repeated, the $\Omega^{m}$ that corresponds to a cylinder becomes infinitely long along its longitudinal direction. In this case, we again do not need to actually solve Eqs. 25-28. It is easy to show

$$
\text { (cylinder) } \bar{D}_{s s}^{m}=\mathbf{a}^{T} D^{0} \mathbf{a}
$$

where $\mathbf{a}$ is the normalized $(\|\mathbf{a}\|=1)$ vector parallel to the axis of the cylinder. So the only compartment where we actually solve Eqs. $25-28$ for $\bar{D}_{s s}^{m}$ is the extra-cellular compartment:

(extra-cellular compartment) $\bar{D}_{s s}^{m}$ from solution of Eqs. 25-28.

We note that the expressions in Eq. 22, Eq. 29, Eq. 30 have been used in previous works (for example, in [43]) for the Karger model. 
The mathematical derivation of the function $c(t)$ from the multiple compartment Bloch-Torrey PDE can be found in [32]. To see this more intuitively, we can look at the ODE satisfied by the integral of the solution of the BlochTorrey PDE in a homogeneous medium with the constant diffusion tensor $\bar{D}^{0}$. It is easy to show for a general gradient time profile $f(s)$, by the use of the Laplace transform, that the integral of the magnetization satisfies, for any point in $[0, T E]$ :

$$
\bar{M}^{h o m}(b, t):=\int_{\mathbb{R}^{3}} m(\mathbf{x}, t \mid \mathbf{g}) d \mathbf{x}=e^{-\mathbf{g}^{T} \bar{D}^{0} \mathbf{g} \gamma^{2} \int_{0}^{t} d u\left(\int_{0}^{u} f(s) d s\right)^{2}}, \quad 0 \leq t \leq T E,
$$

where the term in the exponential contains the b-value, defined for example, in [50]. We note that the above equation is valid for any $t \in[0, T E]$, not just at the echo time $T E$. Then it is easy to show $c(t)$ satisfies

$$
c(t) \equiv\left(\int_{0}^{t} f(s) d s\right)^{2}=\frac{-\frac{\partial \bar{M}^{h o m}(b, t)}{\partial t}}{\mathbf{g}^{T} \bar{D}^{0} \mathbf{g} \gamma^{2} \bar{M}^{h o m}(b, t)},
$$

for any profile $f$.

For the pulsed gradient spin echo (PGSE) sequence[46],

$$
c(t)= \begin{cases}\left(t-t_{1}\right)^{2}, & t_{1} \leq t \leq t_{1}+\delta \\ \delta^{2}, & t_{1}+\delta<t \leq t_{1}+\Delta \\ \left(t-t_{1}-\Delta-\delta\right)^{2}, & t_{1}+\Delta<t \leq t_{1}+\Delta+\delta\end{cases}
$$

In the narrow pulse regime, $\delta \ll \Delta$, we can see that (32) becomes one interval and on that interval we obtain the coefficient $\delta^{2}$ of the Karger model.

The initial condition of the FPK model is the same as for the Karger model, namely, Eq. 17, and the dMRI signal for the FPK model is

$$
S_{F P K}(b)=\sum_{p=1}^{W} \bar{M}_{F P K}^{p}(b, T E) .
$$

The $A D C_{0}$ of the FPK model is

$$
A D C_{0}^{F P K}=\sum_{p=1}^{W} v^{p} \frac{\mathbf{g}^{T} \bar{D}_{s s}^{p} \mathbf{g}}{\|\mathbf{g}\|^{2}} .
$$




\section{Method}

We solved the multiple compartment Bloch-Torrey PDE in the representative volume $C$ containing different cellular configurations using the finite elements method described in [51]. We generated the finite elements mesh in the computational domain $C$ using the mesh generation platform Salome (http://www.salome-platform.org/). The computational domain $C$ contains a configuration of spheres and cylinders. The angles of the cylinders were chosen so that when $C$ is periodically repeated, the cylinders become infinitely long in the longitudinal direction.

We set the intrinsic diffusion coefficient in all the compartments to be $D^{0}=$ $3 \times 10^{-3} \mathrm{~mm}^{2} / \mathrm{s}$. The spin density was set to $\frac{1}{|C|}$ for all the compartments to normalize the dMRI signals to 1 at $b=0$. For simplicity, for the PGSE sequence, we set $t_{1}=0$ and $T E$ to be immediately after the diffusion-encoding sequence.

We used the same macroscopic parameters for both the FPK and Karger models, in other words, the exchange times and the (steady-state) effective diffusion tensors defined by Eq. 22, Eq. 29, Eq. 30, Eq. 31.

We also computed two limit cases. If there is no exchange between any of the compartments the dMRI signal is

$$
S_{N O E X}(b)=\sum_{p=1}^{W} v^{p} \exp \left(-\frac{\mathbf{g}^{T} \bar{D}_{e f f}^{p} \mathbf{g}}{\|\mathbf{g}\|^{2}} b\right) .
$$

If the exchange between the compartments is complete the dMRI signal is:

$$
S_{C O M P E X}(b)=\exp \left(-\left(\sum_{p=1}^{W} v^{p} \frac{\mathbf{g}^{T} \bar{D}_{e f f}^{p} \mathbf{g}}{\|\mathbf{g}\|^{2}}\right) b\right) .
$$

There are many references on the size of the cells found in brain tissue, and these references are listed, for example, in [16]. We chose the sizes of the cells in our simulations to be within the physically reasonable range for the neurites when defining the cylinder compartments and in the range of the neuronal bodies and glial cells when defining the spheres compartments. The cell membrane permeabilities were chosen so the dMRI signal curves fall visually somewhere between the no exchange and the complete exchange curves. 
We computed the dMRI signal at 20 b-values between 0 and $4000 \mathrm{~s} / \mathrm{mm}^{2}$. We will compare the following 5 models of the dMRI signal:

1. "PDE": The (microscopic) multiple compartment Bloch-Torrey PDE.

2. "FPK": The (macroscopic) FPK model.

3. "KAR": The (macroscopic) Karger model.

4. "NO EX": The (macroscopic) No Exchange signal.

5. "COMP EX": The (macroscopic) Complete Exchange signal.

The signals will be labeled as the above in the figures. We also computed the compartment magnetizations, $\bar{M}_{P D E}^{p}(b, t), \bar{M}_{F P K}^{p}(b, t)$ and $\bar{M}_{K A R}^{p}(b, t)$, for the different compartments as a function of time.

The simulations were performed on a Dell PRECISION M4700 workstation (Intel(R) Core(TM)i7 CPU 3740QM@2.70GB). The solution of the microscopic Bloch-Torrey PDE took anywhere between 4 seconds to $37 \mathrm{~min}$ utes, per b-value, depending on the cellular configuration and the diffusionencoding sequence. The memory usage was between 28Mbytes to 409Mbytes. Table 1 shows the details of the simulation time and the memory usage. The FPK model was solved using the Matlab command "ode45". The solution of the ODE took a few seconds per b-value. The memory usage was negligible.

\begin{tabular}{|c|c|c|c|}
\hline \multirow{2}{*}{ Domain } & \multirow{2}{*}{ Memory (NFE) } & \multicolumn{2}{|c|}{ Computational time } \\
\cline { 3 - 4 } & & $\delta=\Delta=5 \mathrm{~ms}$ & $\delta=\Delta=40 \mathrm{~ms}$ \\
\hline 1 Sphere & 36MB (8000) & 15 seconds & 38 seconds \\
\hline 76 spheres & $409 \mathrm{MB}(277000)$ & 15 minutes & 37 minutes \\
\hline Slanted parallel cylinders & $36 \mathrm{MB}(8000)$ & 15 seconds & 37 seconds \\
\hline 3-compartment cylinder & $28 \mathrm{MB}(3000)$ & 4 seconds & 10 seconds \\
\hline Cylinders + spheres & $227 \mathrm{MB}(71000)$ & 8 minutes & 22 minutes \\
\hline
\end{tabular}

Table 1: Memory usage and average computational time per b-value to solve the BlochTorrey PDE for the various simulations. The number in the parenthesis after the memory usage is the number of finite elements (NFE) rounded to the nearest thousand.

\section{Numerical study of the FPK model}

In this section, we will first illustrate the convergence properties of the FPK model on a simple cellular configuration where $C$ contains a single sphere. Then we put more complex cellular configurations inside $C$. 


\subsection{Periodic lattice of spheres}

To show the convergence of the FPK model signal to the reference signal, we put a single sphere inside the representative volume $C=\left[-\frac{l}{2}, \frac{l}{2}\right]$. From the point of view of the FPK model, this domain contains two compartments: the extra-cellular compartment $\Omega^{e}$ and the sphere compartment $\Omega^{s}$. The radius of the sphere was chosen to correspond to volume fractions of $v^{e}=0.51$ and $v^{s}=0.49$.

The effective diffusion tensor of $\Omega^{s}$ is $\bar{D}^{s}=0$. We computed the steady-state effective diffusion tensor of $\Omega^{e}$ from Eq. 24 to be:

$$
\bar{D}_{s s}^{e}=\left[\begin{array}{lll}
2.32 & 0.00 & 0.00 \\
0.00 & 2.32 & 0.00 \\
0.00 & 0.00 & 2.32
\end{array}\right] \times 10^{-3} \mathrm{~mm}^{2} / s .
$$

This value is independent of $l$.

We defined the macroscopic length to be:

$$
L \equiv \sqrt{2 \max _{p \in\{s, e\}}\left(\mathbf{g}^{T} \bar{D}_{s s}^{p} \mathbf{g}\right)(\Delta+\delta)},
$$

the mean squared displacement of the fastest diffusing compartment in the diffusion-encoding direction at the steady-state value during the time interval $[0, \Delta+\delta]$.

The scaling of the permeability coefficient in the derivation of the FPK model is linear in $\varepsilon=\frac{l}{L}$, and we chose to scale $\kappa$ with $l$ :

1. $l=2.5 \mu \mathrm{m}, \kappa=\kappa_{0}$,

2. $l=5 \mu \mathrm{m}, \kappa=2 \kappa_{0}$,

3. $l=10 \mu \mathrm{m}, \kappa=4 \kappa_{0}$,

4. $l=20 \mu \mathrm{m}, \kappa=8 \kappa_{0}$,

for four different values of $l$, while fixing $\kappa_{0}$ as well as the $\delta$ and $\Delta$ of the PGSE sequence. For this set of four $l$ 's, we simulated three values of $\kappa_{0}$ :

1. $\kappa_{0}=0.5 \times 10^{-5} \mathrm{~m} / \mathrm{s}$ (labeled "low $\kappa$ "),

2. $\kappa_{0}=1 \times 10^{-5} \mathrm{~m} / \mathrm{s}$ (labeled "mid $\left.\kappa "\right)$,

3. $\kappa_{0}=2 \times 10^{-5} \mathrm{~m} / \mathrm{s}$ (labeled "high $\kappa$ "),

and three PGSE sequences (two narrow-pulse, and one non-narrow pulse): 
1. $\delta=3 \mathrm{~ms}, \Delta=40 \mathrm{~ms}$,

2. $\delta=3 \mathrm{~ms}, \Delta=80 \mathrm{~ms}$,

3. $\delta=40 \mathrm{~ms}, \Delta=40 \mathrm{~ms}$.

In Fig. 1(a) we show the convergence of $\left|S_{P D E}(b)-S_{F P K}(b)\right|$ with the nondimensional parameter,

$$
\varepsilon:=l / L
$$

for a range of $\kappa_{0}, \delta$ and $\Delta$, at a single b-value, $b=4000 \mathrm{~s} / \mathrm{mm}^{2}$. We see that the convergence rate is

$$
\left|S_{P D E}(b)-S_{F P K}(b)\right|=O\left(\varepsilon^{\alpha}\right),
$$

where $\alpha$ is about 2. The convergence rate of $\alpha=2$ is quite clear for $\kappa_{0}=$ $0.5 \times 10^{5} \mathrm{~m} / \mathrm{s}$ and $\kappa_{0}=1 \times 10^{5} \mathrm{~m} / \mathrm{s}$ and this rate is not attained at the highest set of permeabilities, $\kappa_{0}=2 \times 10^{5} \mathrm{~m} / \mathrm{s}$. For the non-narrow pulse PGSE sequence, $\delta=40 \mathrm{~ms}, \Delta=40 \mathrm{~ms}$, on $C=[-2.5 \mu \mathrm{m}, 2.5 \mu \mathrm{m}]^{3}, \kappa=10^{-5} \mathrm{~m} / \mathrm{s}$, we see that the $S_{F P K}(b)$ is very close to $S_{P D E}(b)$, whereas $S_{K A R}(b)$ is further away (Fig. 1(b)). We also show the compartment magnetizations in $\Omega^{e}$ and $\Omega^{s}$ from $t=0 \mathrm{~ms}$ to $t=80 \mathrm{~ms}$ at $b=2000 \mathrm{~s} / \mathrm{mm}^{2}$ (Fig. 1(c) and Fig. $1(\mathrm{~d})$ ). We see that $\bar{M}_{F P K}^{e}(2000, t)$ and $\bar{M}_{F P K}^{s}(2000, t)$ follow the time evolution of $\bar{M}_{P D E}^{e}(2000, t)$ and $\bar{M}_{P D E}^{s}(2000, t)$, whereas the Karger model compartment magnetizations $\bar{M}_{K A R}^{e}(2000, t)$ and $\bar{M}_{K A R}^{s}(2000, t)$ do not. 


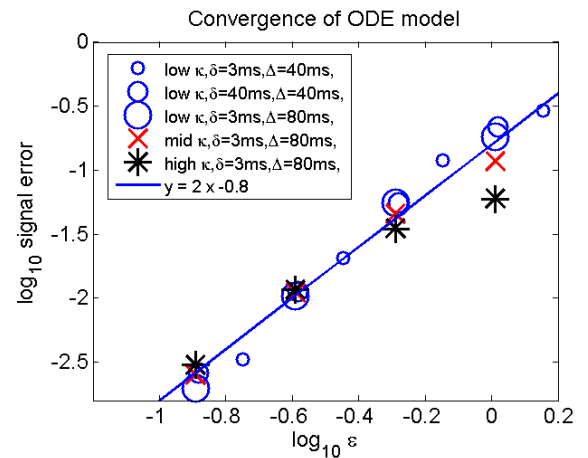

(a) Second order convergence in $\varepsilon, b=$ $4000 \mathrm{~s} / \mathrm{mm}^{2}$.

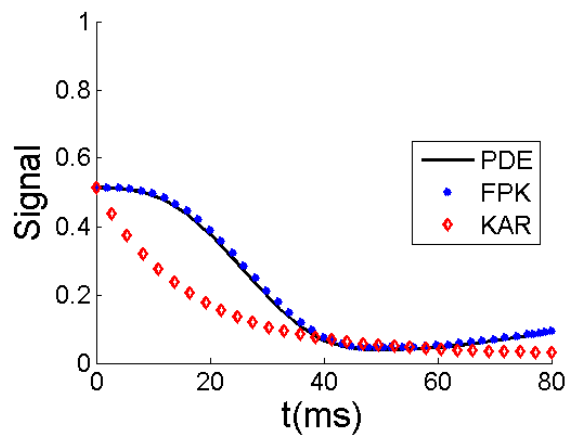

(c) $\bar{M}^{e}(2000, t)$

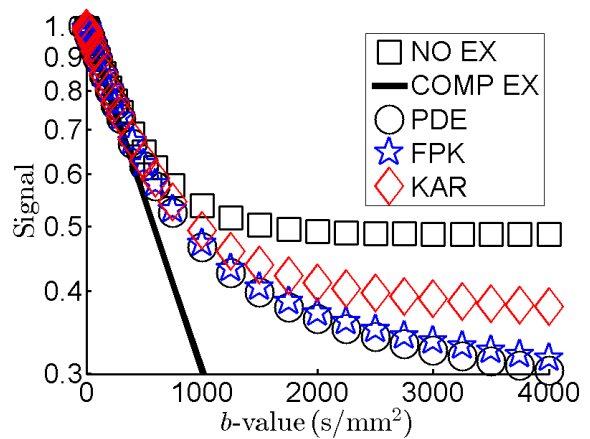

(b) $S(b)$

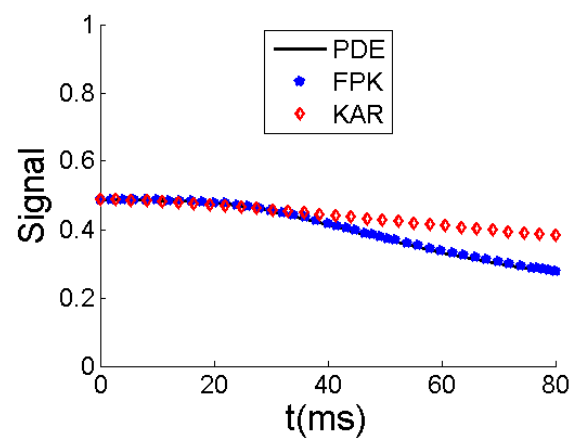

(d) $\bar{M}^{s}(2000, t)$

Figure 1: (a) The FPK model signal converges to the reference signal with second order convergence in $\varepsilon=l / L$, where $l$ is the side length of the representative volume $C$ and $L$ is a measure of the diffusion displacement. (b) DMRI signals: $S_{P D E}(b)$, $S_{F P K}(b), S_{K A R}(b), S_{N O E X}(b), S_{C O M P E X}(b)$. Simulation parameters: sphere radius $R^{s}=2.45 \mu \mathrm{m}, D^{0}=3 \times 10^{-3} \mathrm{~mm}^{2} / \mathrm{s}, \kappa=10^{-5} \mathrm{~m} / \mathrm{s}$, PGSE sequence with $\delta=40 \mathrm{~ms}$, $\Delta=40 \mathrm{~ms}$. (c) Compartment magnetization in the extra-cellular compartment at $b=$ $2000 \mathrm{~s} / \mathrm{mm}^{2}: \bar{M}_{P D E}^{e}(2000, t), \bar{M}_{F P K}^{e}(2000, t), \bar{M}_{K A R}^{e}(2000, t)$, same simulation parameters as b). (d) Compartment magnetization in the sphere compartment at $b=2000 \mathrm{~s} / \mathrm{mm}^{2}$ : $\bar{M}_{P D E}^{s}(2000, t), \bar{M}_{F P K}^{s}(2000, t), \bar{M}_{K A R}^{s}(2000, t)$, same simulation parameters as b).

\subsection{Simulation on complex geometries}

We constructed the representative volume $C=[-5 \mu \mathrm{m}, 5 \mu \mathrm{m}]^{3}$ in Fig. 2(a) containing 76 spherical cells with a range of radii between $0.6 \mu \mathrm{m}$ and $2.55 \mu \mathrm{m}$. In theory one would have to make the 76 spheres 76 different compart- 
ments, each with its volume fraction and surface-to-volume ratio. However, we just combine the 76 spheres to form one compartment $\Omega^{s}$. The extra-cellular space forms a second compartment $\Omega^{e}$. The corresponding volume fractions are $v^{s}=0.65$ and $v^{e}=0.35$. The surface to volume ratio is $\left|\Gamma^{s e}\right| /\left|\Omega^{s}\right|=1.85 \mu \mathrm{m}^{-1}$. We note that we are assuming that $\left|\Gamma^{s e}\right| /\left|\Omega^{s}\right|$ gives a good approximation of the average surface-to-volume ratios of all the 76 spheres. Certainly, the approximation is exact if all the spheres have the same radii. We then computed

$$
\bar{D}^{e}=\left[\begin{array}{lll}
2.20 & 0.00 & 0.00 \\
0.00 & 2.25 & 0.00 \\
0.00 & 0.00 & 2.24
\end{array}\right] \times 10^{-3} \mathrm{~mm}^{2} / \mathrm{s} .
$$

We see in Fig. 2(b) that using the two-compartment FPK model still gives a good approximation to the signal of the full PDE if we use the average surface-to-volume ratio of the 76 spheres. The homogenization parameter $\varepsilon=$ $10 \mu \mathrm{m} / \sqrt{2 \times 2.2 \times 10^{-3} \mathrm{~mm}^{2} / \mathrm{s} \times(50 \mathrm{~ms})}=0.67$, translating into a diffusion displacement that is 1.5 times the side length of $C$.

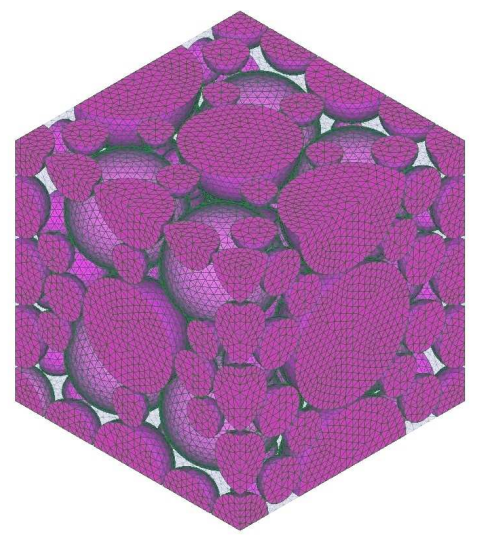

(a) Finite elements mesh of $C$

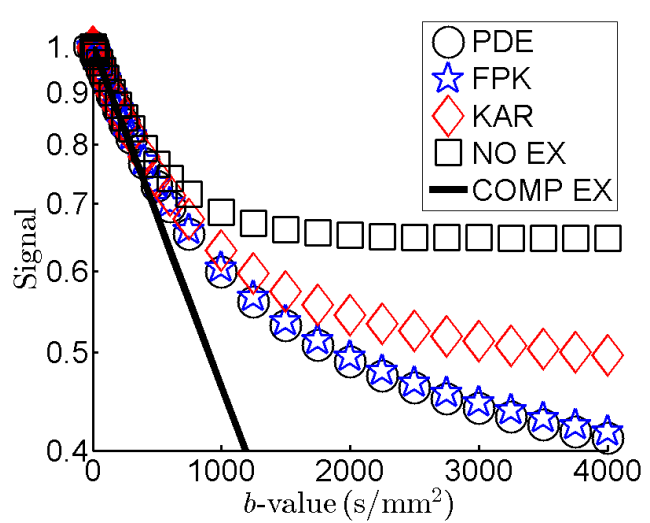

(b) DMRI signals

Figure 2: (a) The computational domain $C=[-5 \mu \mathrm{m}, 5 \mu \mathrm{m}]^{3}$ contains 76 spheres with radii between $0.6 \mu \mathrm{m}$ and $2.55 \mu \mathrm{m}$. The volume fraction of the spheres is $v^{s}=0.65$, and of the extra-cellular space is $v^{e}=0.35$. We set $\kappa=10^{-5} \mathrm{~m} / \mathrm{s}, D^{0}=3 \times 10^{-3} \mathrm{~mm}^{2} / \mathrm{s}$. (b) The dMRI signals: $S_{P D E}(b), S_{F P K}(b), S_{K A R}(b), S_{N O E X}(b), S_{C O M P E X}(b)$. The diffusion-encoding sequence is PGSE: $\delta=\Delta=25 \mathrm{~ms}$.

Now, to test whether the FPK model works properly for anisotropic diffu- 
sion, we construct a computational domain $C=[-2.89,2.89] \times[-2.5,2.5] \times$ $[-5,5] \mu \mathrm{m}^{3}$ containing slanted cylinders that all lie parallel to $\mathbf{g}_{1}=(0.5,0,0.87)$ and have a radius of $R^{c}=2.35 \mu \mathrm{m}$ (Fig. 3(a)). We consider the domain to be made up of two compartments, one is $\Omega^{c}$, the compartment of all the cylinders, $v^{c}=0.69$, the other is the extra-cellular space, $\Omega^{e}, v^{e}=0.31$. The volumes and surface areas are $\left|\Omega^{e}\right|=91 \mu \mathrm{m}^{3},\left|\Omega^{c}\right|=198 \mu \mathrm{m}^{3}$, and $\left|\Gamma^{c e}\right|=170 \mu \mathrm{m}^{2}$. We computed from Eq. 30:

$$
\bar{D}^{c}=\left[\begin{array}{lll}
0.75 & 0.00 & 1.30 \\
0.00 & 0.00 & 0.00 \\
1.30 & 0.00 & 2.25
\end{array}\right] \times 10^{-3} \mathrm{~mm}^{2} / \mathrm{s}
$$

and from Eq. 24:

$$
\bar{D}^{e}=\left[\begin{array}{lll}
1.88 & 0.00 & 0.65 \\
0.00 & 2.52 & 0.00 \\
0.65 & 0.00 & 2.63
\end{array}\right] \times 10^{-3} \mathrm{~mm}^{2} / \mathrm{s}
$$

We set $\kappa=10^{-5} \mathrm{~m} / \mathrm{s}$, and for the PGSE sequence, $\delta=\Delta=80 \mathrm{~ms}$. The simulation was done for three different gradient directions (parallel, perpendicular and 45 degrees from axes of the cylinders). We show in Fig. 3(b) that $S_{P D E}(b)$ and $S_{F P K}(b)$ are close in all three gradient directions. In the direction perpendicular to the cyinders, the homogenization parameter is $\varepsilon=0.23$, corresponding to a diffusion displacement that is 4.4 times the distance between cylinder centers. 


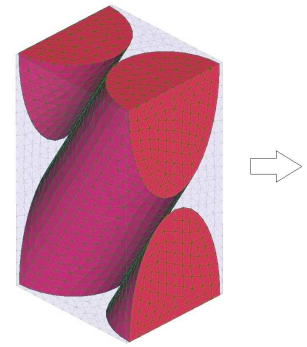

(a) Left. mesh of $C$. Right. periodic extension.

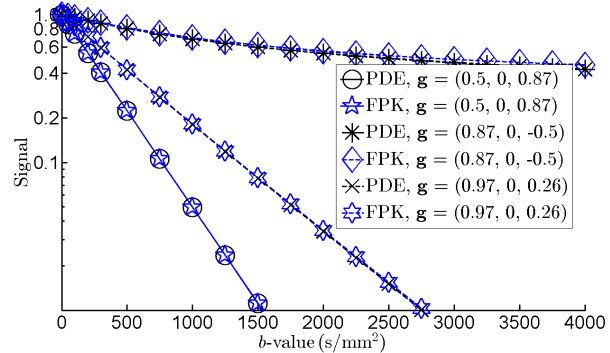

(b) DMRI signals

Figure 3: (a) Left: finite elements mesh of $C=[-2.89,2.89] \times[-2.5,2.5] \times[-5,5] \mu \mathrm{m}^{3}$. Right: when periodically extended, the cylinders are infinitely long. (b): The signals $S_{P D E}(b)$ and $S_{F P K}(b)$. Simulation parameters: $\kappa=10^{-5} \mathrm{~m} / \mathrm{s}, D^{0}=3 \times 10^{-3} \mathrm{~mm}^{2} / \mathrm{s}$; the sequence is PGSE, $\delta=\Delta=80 \mathrm{~ms}$; the cylinders have radius $R^{c}=2.35 \mu \mathrm{m}$. The gradient directions are $\mathbf{g}_{1}=(0.5,0,0.87)$ and $\mathbf{g}_{2}=(0.87,0,-0.5)$, corresponding to parallel and perpendicular directions to the axis of the cylinders, respectively, and $\mathbf{g}_{3}=(0.97,0,0.26)$, lying in the middle of $\mathbf{g}_{1}$ and $\mathbf{g}_{2}$.

Next, we constructed a computational domain $C=[-2.75,2.75]^{2} \times[-0.5,0.5] \mu \mathrm{m}^{3}$ with a cell membrane compartment (Fig. 4(a)). The computational domain $C$ contains a cylinder $\left(R^{c}=2.0 \mu \mathrm{m}\right)$ with a thick membrane layer (thickness $h=0.45 \mu \mathrm{m}$ ) outside it. We consider $C$ to be made up of three compartments, the extra-cellular space $\Omega^{e}\left(\left|\Omega^{e}\right|=11.5 \mu \mathrm{m}^{3}\right)$, the membrane compartment $\Omega^{m}\left(\left|\Omega^{m}\right|=6.3 \mu \mathrm{m}^{3}\right)$, and the cylinder compartment $\Omega^{c}\left(\left|\Omega^{c}\right|=12.5 \mu \mathrm{m}^{3}\right)$, with the corresponding surfaces $\Gamma^{e m}\left(\left|\Gamma^{e m}\right|=15.4 \mu \mathrm{m}^{2}\right)$ and $\Gamma^{m c}\left(\left|\Gamma^{m c}\right|=12.5 \mu \mathrm{m}^{2}\right)\left(\left|\Gamma^{e c}\right|=0\right.$, obviously $)$. We know $\bar{D}^{c}$ and $\bar{D}^{m}$ from Eq. 30 and Eq. 29. We computed

$$
\bar{D}^{e}=\left[\begin{array}{lll}
1.70 & 0.00 & 0.00 \\
0.00 & 1.70 & 0.00 \\
0.00 & 0.00 & 3.00
\end{array}\right] \times 10^{-3} \mathrm{~mm}^{2} / \mathrm{s} \text {. }
$$

We would like to know if the membrane compartment can be detected in the dMRI signal. For this reason, we constructed a second computational domain $C$, with two compartments: a combined cylinder and membrane compartment, $\Omega^{m+c}$, with no impedence to diffusion between $\Omega^{m}$ and $\Omega^{c}$ $\left(\left|\Omega^{m+c}\right|=18.8 \mu \mathrm{m}^{3}\right)$, and the same extra-cellular compartment $\Omega^{e}$ as the three compartment domain. In the two compartment domain, there is only one surface $\Gamma^{e(m+c)}\left(\left|\Gamma^{e(m+c)}\right|=15.4 \mu \mathrm{m}^{2}\right)$. 
With permeability $\kappa^{m e}=10^{-5} \mathrm{~m} / \mathrm{s}$, Fig. 4(b) shows $S_{P D E}(b)$ and $S_{F P K}(b)$ in the gradient direction perpendicular with the cylinder axis for both domains. $S_{P D E}(b)$ and $S_{F P K}(b)$ are close to each other in both cases. The difference in the signals from the two domains can be seen in the Fig. 4(b) but it is not clear whether the difference is large enough to be useful in detecting the membrane compartment in experimental settings. The homogenization parameter is $\varepsilon=0.24$ for both cases.

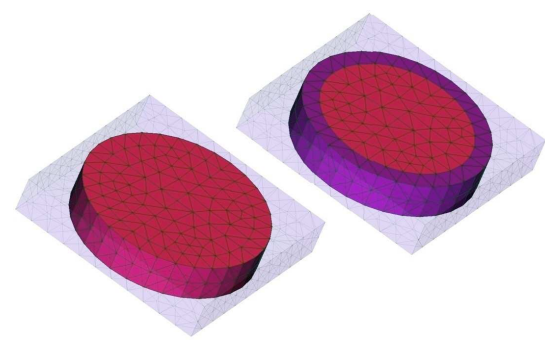

(a) Two and three compartment domains.

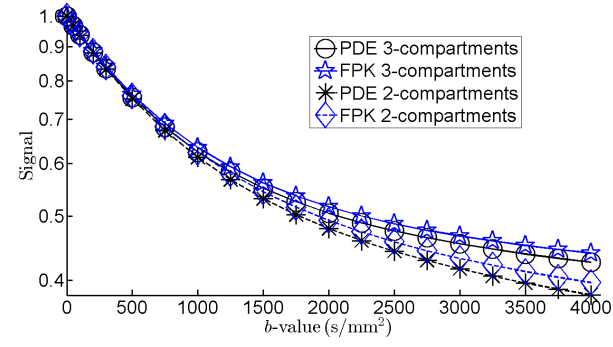

(b) DMRI signals

Figure 4: (a) Finite elements meshes. Left: a two compartment domain $\left(\Omega^{e}\right.$ and $\left.\Omega^{c+m}\right)$. Right: a three compartment domain $\left(\Omega^{e}\right.$ and $\Omega^{c}$ and $\left.\Omega^{m}\right)$. (b) The signals $S_{P D E}(b)$ and $S_{F P K}(b)$ of the 3-compartments domain and the 2-compartments domain. Simulation parameters: $C=[-2.75,2.75]^{2} \times[-0.5,0.5] \mu \mathrm{m}^{3}$; the cylinder has radius $R^{c}=2.0 \mu \mathrm{m}$, the membrane layer has thickness $h=0.45 \mu \mathrm{m}$; the two compartment domain combines the cylinder and the membrane layer compartments into one; $D^{0}=3 \times 10^{-3} \mathrm{~mm}^{2} / \mathrm{s}$, $\kappa=10^{-5} \mathrm{~m} / \mathrm{s}$, the sequence is PGSE, $\delta=\Delta=80 \mathrm{~ms}$, in the gradient direction $\mathbf{g}=(1,0,0)$, perpendicular to the axis of cylinder.

Finally, we constructed a domain of 5 layers of cylinders lying parallel to the $x-y$ plane and 4 layers of spherical cells, all embedded in the extracellular space, see Fig 5(a). The five layers of cylindrical cells are composed of parallel cylinders that are oriented at 0, 26.5, 45, 63.5 and 90 degrees, respectively, from the $x$-axis (cylinder radius $R^{c}=1.0 \mu \mathrm{m}$ ). The 4 layers of 16 spherical cells (sphere radius $R^{s}=1.375 \mu \mathrm{m}$ ) are inserted between the 5 layers of cylindrical cells. When the spheres overlapped with the cylinders, the latter were deformed slightly. In particular, some spheres and cylinders have joint interfaces. Because the cylinders are only slighlty deformed, we still use Eq. (30) for computing the effective diffusion tensor. 
In summary, we consider this domain to have 7 compartments: the extracellular space $\left(\Omega^{e}\right)$, the compartment of the 4 layers of 16 spherical cells $\left(\Omega^{s}\right)$, and 5 compartments consisting of the cylinders with the same orientation $\left(\Omega^{c_{1}}, \cdots, \Omega^{c_{5}}\right)$. In each layer of cylinders, the orientations of the cylinders are the same. We note that the spheres can be combined into one compartment because they have the same effective diffusion tensor (the zero tensor). On the other hand, the effective diffusion tensors of the 5 layers of cylinders are not the same due to their differing orientations, so each layer needs to be considered as a separate compartment.

For the 7 compartments, the volumes are: $\left|\Omega^{e}\right|=383 \mu \mathrm{m}^{3},\left|\Omega^{s}\right|=167 \mu \mathrm{m}^{3}$, $\left|\Omega^{c_{1}}\right|=46 \mu \mathrm{m}^{3},\left|\Omega^{c_{2}}\right|=47 \mu \mathrm{m}^{3},\left|\Omega^{c_{3}}\right|=55 \mu \mathrm{m}^{3},\left|\Omega^{c_{4}}\right|=47 \mu \mathrm{m}^{3}$ and $\left|\Omega^{c_{5}}\right|=$ $46 \mu \mathrm{m}^{3}$. The surface areas are: $\left|\Gamma^{e s}\right|=291 \mu \mathrm{m}^{2},\left|\Gamma^{e c_{1}}\right|=93 \mu \mathrm{m}^{2},\left|\Gamma^{e c_{2}}\right|=$ $85 \mu \mathrm{m}^{2},\left|\Gamma^{e c_{3}}\right|=98 \mu \mathrm{m}^{2},\left|\Gamma^{e c_{4}}\right|=85 \mu \mathrm{m}^{2},\left|\Gamma^{e c_{5}}\right|=93 \mu \mathrm{m}^{2},\left|\Gamma^{s c_{1}}\right|=0,\left|\Gamma^{s c_{2}}\right|=$ $21 \mu \mathrm{m}^{2},\left|\Gamma^{s c_{3}}\right|=37 \mu \mathrm{m}^{2},\left|\Gamma^{s c_{4}}\right|=21 \mu \mathrm{m}^{2},\left|\Gamma^{s c_{5}}\right|=0$. The cylinders have different volumes and surface areas because they are deformed differently by the intervening spheres. The cellular permeability for both cylinders and spheres is set to $\kappa=10^{-5} \mathrm{~m} / \mathrm{s}$. The computational domain is $C=$ $[-3.75,3.75] \times[-7.03,7.03] \times[-3.75,3.75] \mu \mathrm{m}^{3}$. The computed effective extracellular diffusion tensor is:

$$
\bar{D}^{e}=\left[\begin{array}{lll}
2.29 & 0.00 & 0.11 \\
0.00 & 2.05 & 0.00 \\
0.11 & 0.00 & 2.29
\end{array}\right] \times 10^{-3} \mathrm{~mm}^{2} / \mathrm{s}
$$

We computed the signals $S_{P D E}(b)$ and $S_{F P K}(b)$ in three gradient directions: $\mathbf{g}_{1}=(1,0,0), \mathbf{g}_{2}=(1,0,1)$, and $\mathbf{g}_{3}=(1,1,1)$, for the PGSE sequence with $\delta=\Delta=40 \mathrm{~ms}$. Figure 5(b) shows that the FPK and the PDE signals are almost indistinguishable. In the direction $\mathbf{g}_{1}=(1,0,0)$, the homogenization parameter is $\varepsilon=7.5 \mu \mathrm{m} / \sqrt{2 \times 2.29 \times 10^{-3} \mathrm{~mm}^{2} / \mathrm{s} \times 80 \mathrm{~ms}}=0.39$. 


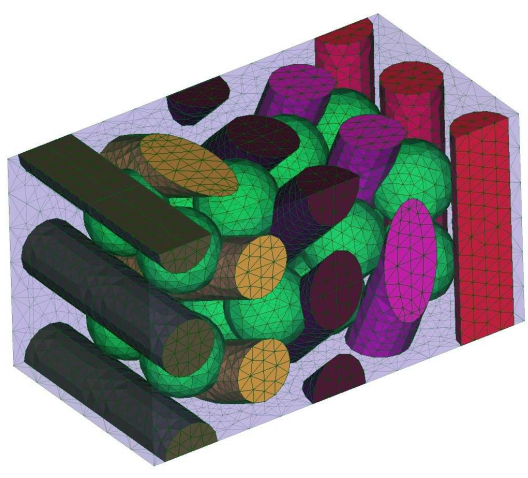

(a) Finite elements mesh of $C$.

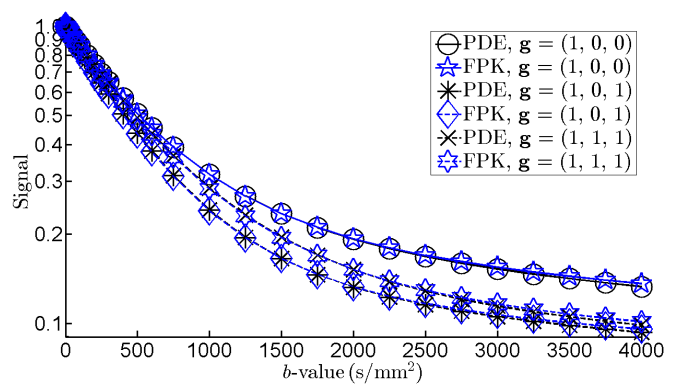

(b) DMRI signals

Figure 5: (a) Finite elements mesh of $C=[-3.75,3.75] \times[-7.03,7.03] \times[-3.75,3.75] \mu \mathrm{m}^{3}$ containing 5 layers of cylinders and 4 layers of spheres. In each layer, the cylinders have the same orientation. (b) The signals $S_{P D E}(b)$ and $S_{F P K}(b)$. Simulation parameters: $D^{0}=$ $3 \times 10^{-3} \mathrm{~mm}^{2} / \mathrm{s}, \kappa=10^{-5} \mathrm{~m} / \mathrm{s}$; the gradient directions are $\mathbf{g}_{1}=(1,0,0), \mathbf{g}_{2}=(1,0,1)$, and $\mathbf{g}_{3}=(1,1,1)$; the pulse sequence is PGSE, with $\delta=\Delta=40 \mathrm{~ms}$.

\section{Preliminary results of applying the FPK model to voxel level dMRI signal}

In this section, we show some preliminary numerical results in two dimensions of applying the FPK model to the voxel level dMRI signal. We construct a two dimensional voxel $V=[-500 \mu \mathrm{m}, 500 \mu \mathrm{m}]^{2}$ by placing 14000 circular biological cells in $V$ whose centers are uniformly randomly distributed in $V$. The radii of the circles were chosen randomly with uniform distribution between 1 and $5 \mu \mathrm{m}$. The cellular volume fraction is 0.42. See Figure 6(a) for the voxel $V$. We will assume that $V$ is bordered by neighboring voxels that are identical to $V$. Since the dMRI diffusion displacement is much smaller than the side length of $V$, this assumption does not limit the generality of this example. We set the intrinsic diffusion coefficient to be $3 \times 10^{-3} \mathrm{~mm}^{2} / \mathrm{s}$ inside and outside the biological cells. and the diffusion-encoding direction to be $\mathbf{g}=[1,0]$.

We set up the FPK model using 2 compartments, one is the ensemble of cells, $\Omega^{c}$, one is the extra-cellular space, $\Omega^{e}$. The total circumference of all the circles is $\Gamma=263550 \mu \mathrm{m}$. The total volume is $1 \mathrm{~mm}^{2}$.

We will attempt to apply the FPK model to this example for two PGSE 
sequences: $\delta=3 \mathrm{~ms}$ and $\Delta=17 \mathrm{~ms}, \delta=3 \mathrm{~ms}$ and $\Delta=77 \mathrm{~ms}$. First we test if the diffusion is approximately Gaussian in the extra-cellular space by solving the Bloch-Torrey PDE where water molecules are placed initially only inside $\Omega^{e}$ and setting $\kappa=0$ to prevent the water molecules from ever entering $\Omega^{c}$. We show in Figure 6(b) the resulting dMRI signal contributions from $\Omega^{e}$, for the two PGSE sequences: $\delta=3 \mathrm{~ms}, \Delta=17 \mathrm{~ms}$, and $\delta=3 \mathrm{~ms}, \Delta=77 \mathrm{~ms}$. It is clear for both sequences, the diffusion is not Gaussian in $\Omega^{e}$. We computed the slope of the log of the signals to obtain a slope of $1.6 \times 10^{-3} \mathrm{~mm}^{2} / \mathrm{s}$ for $\delta=3 \mathrm{~ms}, \Delta=17 \mathrm{~ms}$, and a slope of $1.3 \times 10^{-3} \mathrm{~mm}^{2} / \mathrm{s}$ for $\delta=3 \mathrm{~ms}, \Delta=77 \mathrm{~ms}$. In the same Figure 6(b) we also draw the Gaussian approximations with these two values of the slope. We can see that the signals derivate from the Gaussian approximations already at $b=1000 \mathrm{~s} / \mathrm{mm}^{2}$.

Given that the diffusion is not Gaussian in $\Omega^{e}$ for the two sequences, we ask now what happens if we set $\mathbf{g}^{T} \bar{D}_{e f f}^{e} \mathbf{g}$ in the FPK model to be the slopes we computed in Figure 6(b): $1.6 \times 10^{-3} \mathrm{~mm}^{2} / \mathrm{s}$ for $\delta=3 \mathrm{~ms}, \Delta=17 \mathrm{~ms}$, and a slope of $1.3 \times 10^{-3} \mathrm{~mm}^{2} / \mathrm{s}$ for $\delta=3 \mathrm{~ms}, \Delta=77 \mathrm{~ms}$. We solve the Bloch-Torrey PDE while placing water molecules in both $\Omega^{e}$ and $\Omega^{e}$. To only measure the effect of the non-Gaussianness, without the effect of intercompartment exchange, we set the $\kappa=0$. Thus, the FPK signal is actually the no-exchange limit for this case. We show the results in Figure 6(c). We see, as expected, the PDE signals have more curvature than the no-exchange limit FPK signals, due to the non-Gaussianness of $\Omega^{e}$. We note that this extra curvature of the signal is not related to the inter-compartment exchange because we set $\kappa=0$ for both the PDE and the FPK signals.

Given that the dMRI displacement is between $8-25 \mu \mathrm{m}$, it is possible that a voxel of brain tissue cannot be considered macroscopically homogeneous in the various tissue compartments. We showed in this section an example of a non-Gaussian extra-cellular compartment. In addition, if a dendrite tree of a neuron is to be considered a diffusion compartment in a macroscopic model, it is possible that the tree structure may cause non-Gaussianness at physically relevant dMRI diffusion times. Thus, we believe a more general macroscopic model that allows some form of non-Gaussianness in the various compartments while still allowing inter-compartment exchange to be a useful direction of future work. It is possible that such a model may be obtained by considering a mathematical homogenization approach that was used obtain the FPK model. 


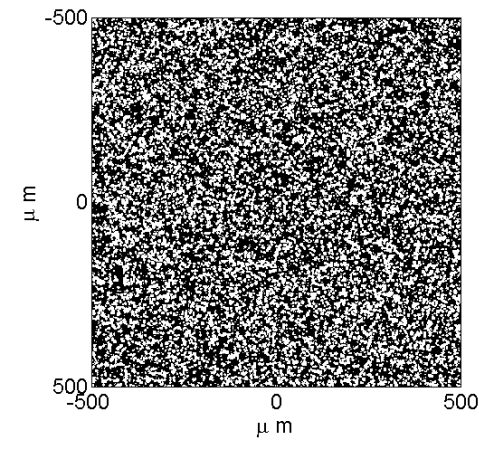

(a) A voxel in two dimensions

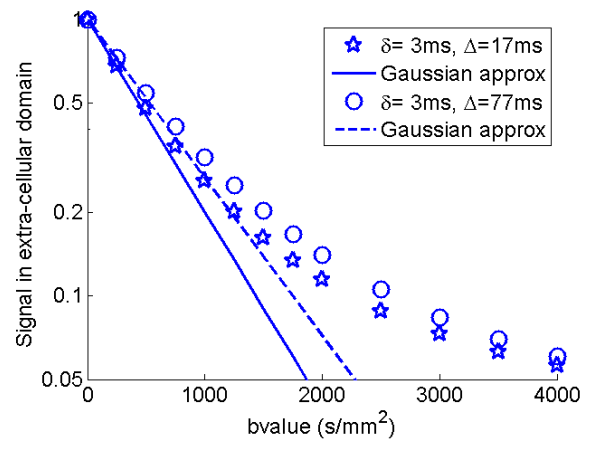

(b) From extra-cellular domain

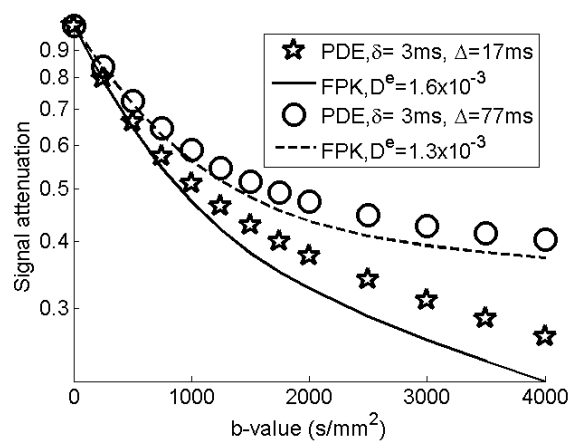

(c) DMRI Signal

Figure 6: (a) The voxel is $V=[-500 \mu \mathrm{m}, 500 \mu \mathrm{m}]$. There are 14000 circular biological cells in $V$ whose centers are uniformly randomly distributed in $V$. The radii of the circles were chosen randomly with uniform distribution between 1 and $5 \mu \mathrm{m}$. The cellular volume fraction is 0.42. (b) The dMRI signal contributions from the extra-cellular space, $\Omega^{e}$, only, for two PGSE sequences: $\delta=3 \mathrm{~ms}, \Delta=17 \mathrm{~ms}$, and $\delta=3 \mathrm{~ms}, \Delta=77 \mathrm{~ms}$. The permeability is set to $\kappa=0 \mathrm{~m} / \mathrm{s}$ to stop the water molecules, initially placed in $\Omega^{e}$ only, from ever entering $\Omega^{e}$. The intrinsic diffusion coefficient is $D^{0}=3 \times 10^{-3} \mathrm{~mm}^{2} / \mathrm{s}$. The signal contributions are not Gaussian after $b=1000 \mathrm{~s} / \mathrm{mm}^{2}$. We computed the slopes of the signals to be $1.6 \times 10^{-3} \mathrm{~mm}^{2} / \mathrm{s}$ for $\delta=3 \mathrm{~ms}, \Delta=17 \mathrm{~ms}$, and $1.3 \times 10^{-3} \mathrm{~mm}^{2} / \mathrm{s}$ for $\delta=3 \mathrm{~ms}, \Delta=77 \mathrm{~ms}$. We include the Gaussian approximations of the signals using these slopes on the plot. (c) The dMRI signals from the entire voxel $V$ for the PGSE sequences, $\delta=3 \mathrm{~ms}, \Delta=17 \mathrm{~ms}$, and $\delta=3 \mathrm{~ms}, \Delta=77 \mathrm{~ms}$. The permeability is set to $\kappa=0 \mathrm{~m} / \mathrm{s}$ and water molecules, initially placed in both $\Omega^{e}$ and $\Omega^{c}$, do not moved between $\Omega^{e}$ and $\Omega^{c}$. The PDE signal shows more curvature than the FPK signal for both PGSE sequences due to the non-Gaussianness of the diffusion in $\Omega^{e}$. The FPK signal were obtained by setting $\mathbf{g}^{T} \bar{D}_{\text {eff }}^{e} \mathbf{g}$ to be the slopes we computed in Figure $6(\mathrm{~b}): 1.6 \times 10^{-3} \mathrm{~mm}^{2} / \mathrm{s}$ for $\delta=3 \mathrm{~ms}$, $\Delta=17 \mathrm{~ms}$, and $1.3 \times 10^{-3} \mathrm{~mm}^{2} / \mathrm{s}$ for $\delta=3 \mathrm{~ms}, \Delta=77 \mathrm{~ms}$. 


\section{Conclusions}

We conducted a numerical study of the macroscopic Homogenized ODE (FPK) model of the dMRI signal in voxels made up of periodic copies of a representative volume $C$ that contains spheres and long cylinders of various sizes and orientations. We showed numerically that the FPK signal approaches the reference signal computed by solving the Bloch-Torrey equation in $O\left(\varepsilon^{2}\right)$, where $\varepsilon$ is the ratio between the size of the representative volume and a measure of the diffusion length. We also show that when the narrow pulse assumption is not satisfied, the FPK model offers a much better approximation of the reference signal than the Karger model. Finaly we showed preliminary results of applying the FPK model to a voxel that is not made up of periodic copies of a representative volume and discussed potential issues that may arise. More work remains to be done to improve macroscopic dMRI signal models so that they are applicable to brain tissue dMRI.

\section{Appendix}

The derivation of the finite pulse Karger model[32] starts with the multiple compartment Bloch-Torrey PDE (Eqs. 1-7) and uses periodic homogenization theory [49], where the voxel was assumed to be a periodic repetition of a representative volume $C$ The magnetization is written as a separate two-scale asymptotic expansion, in $\mathbf{x}$ and $\mathbf{x} / \varepsilon$, in each compartment $p$,

$$
\begin{gathered}
M^{1}(\mathbf{x}, t \mid \mathbf{g})=M_{0}^{1}\left(\mathbf{x}, \frac{\mathbf{x}}{\varepsilon}, t \mid \mathbf{g}\right)+\varepsilon M_{1}^{1}\left(\mathbf{x}, \frac{\mathbf{x}}{\varepsilon}, t \mid \mathbf{g}\right)+\varepsilon^{2} M_{2}^{1}\left(\mathbf{x}, \frac{\mathbf{x}}{\varepsilon}, t \mid \mathbf{g}\right)+\cdots \\
\vdots \\
M^{p}(\mathbf{x}, t \mid \mathbf{g})=M_{0}^{p}\left(\mathbf{x}, \frac{\mathbf{x}}{\varepsilon}, t \mid \mathbf{g}\right)+\varepsilon M_{1}^{p}\left(\mathbf{x}, \frac{\mathbf{x}}{\varepsilon}, t \mid \mathbf{g}\right)+\varepsilon^{2} M_{2}^{p}\left(\mathbf{x}, \frac{\mathbf{x}}{\varepsilon}, t \mid \mathbf{g}\right)+\cdots \\
\vdots \\
M^{W}(\mathbf{x}, t \mid \mathbf{g})=M_{0}^{W}\left(\mathbf{x}, \frac{\mathbf{x}}{\varepsilon}, t \mid \mathbf{g}\right)+\varepsilon M_{1}^{W}\left(\mathbf{x}, \frac{\mathbf{x}}{\varepsilon}, t \mid \mathbf{g}\right)+\varepsilon^{2} M_{2}^{W}\left(\mathbf{x}, \frac{\mathbf{x}}{\varepsilon}, t \mid \mathbf{g}\right)+\cdots
\end{gathered}
$$

where $\mathbf{x}$ is the macroscopic spatial variable and $\mathbf{x} / \varepsilon$ is the microscopic spatial variable. The homogenization scaling parameter is $\varepsilon=l / L$, where $l$ is the side length of $C$ and $L$ is the macroscopic length scale. 
The idea of homogenization is that, assuming $\varepsilon$ is small, the mean value of the order zero term in $\varepsilon, M_{0}^{p}$, with respect to microscopic variable would provide an approximation of $M^{p}$. By matching terms in powers of $\varepsilon$, one can derive a problem for these mean values where only the macroscopic variable $\mathbf{x}$ appears. Before proceeding with the asymptotic matching, the scaling of the permeability coefficient $\kappa$ with $\varepsilon$ must be chosen. The relevant scaling to obtain the FPK model is:

$$
\kappa=O\left(\frac{\sigma}{\mu} \varepsilon\right)
$$

where $\sigma$ (in the cell membranes) has the unit of diffusivity and $\mu$ (width in the cell membranes) has the unit of length. Other choices of the behavior of $\kappa$ as a function of $\varepsilon$, for example,

$$
\kappa=O\left(\frac{\sigma}{\mu} \varepsilon^{m}\right), m \neq 1
$$

can be made and will give different macroscopic models. This is the subject of future work.

\section{Acknowledgment}

This work was funded in part by the Agence Nationale de la Recherche project "SIMUDMRI".

\section{References}

[1] D. Le Bihan, E. Breton, D. Lallemand, P. Grenier, E. Cabanis, M. LavalJeantet, MR imaging of intravoxel incoherent motions: application to diffusion and perfusion in neurologic disorders., Radiology 161 (1986) 401-407.

[2] M. Moseley, J. Kucharczyk, J. Mintorovitch, Y. Cohen, J. Kurhanewicz, N. Derugin, H. Asgari, D. Norman, Diffusion-weighted MR imaging of acute stroke: correlation with T2- weighted and magnetic susceptibilityenhanced MR imaging in cats, AJNR Am J Neuroradiol 11 (1990) 423429.

[3] S. Warach, D. Chien, W. Li, M. Ronthal, R. R. Edelman, Fast magnetic resonance diffusion-weighted imaging of acute human stroke, Neurology 42 (1992) 1717-. 
[4] T. Sugahara, Y. Korogi, M. Kochi, I. Ikushima, Y. Shigematu, T. Hirai, T. Okuda, L. Liang, Y. Ge, Y. Komohara, Y. Ushio, M. Takahashi, Usefulness of diffusion-weighted MRI with echo-planar technique in the evaluation of cellularity in gliomas, J. Magn. Reson. Imaging 9 (1999) $53-60$.

[5] Y. Tsushima, A. Takahashi-Taketomi, K. Endo, Magnetic resonance (MR) differential diagnosis of breast tumors using apparent diffusion coefficient (adc) on 1.5-t, J. Magn. Reson. Imaging 30 (2009) 249-255.

[6] S. E. Maier, Y. Sun, R. V. Mulkern, Diffusion imaging of brain tumors, NMR Biomed. 23 (2010) 849-864.

[7] D. Le Bihan, H. Johansen-Berg, Diffusion MRI at 25: Exploring brain tissue structure and function, NeuroImage 61 (2012) 324-341.

[8] M. Lazar, Mapping brain anatomical connectivity using white matter tractography, NMR Biomed. 23 (2010) 821-835.

[9] D. LeBihan, S.-i. Urayama, T. Aso, T. Hanakawa, H. Fukuyama, Direct and fast detection of neuronal activation in the human brain with diffusion mri, PNAS 103 (2006) 8263-8268.

[10] J. D. Quirk, G. L. Bretthorst, T. Q. Duong, A. Z. Snyder, C. S. Springer, J. J. Ackerman, J. J. Neil, Equilibrium water exchange between the intra- and extracellular spaces of mammalian brain, Magn. Reson. Med. 50 (2003) 493-499.

[11] H. Cheng, S. Torquato, Effective conductivity of periodic arrays of spheres with interfacial resistance, Proceedings: Mathematical, Physical and Engineering Sciences 453 (1997) 145-161.

[12] D. Hasselman, L. F. Johnson, Effective thermal conductivity of composites with interfacial thermal barrier resistance, Journal of Composite Materials 21 (1987) 508-515.

[13] L. L. Latour, K. Svoboda, P. P. Mitra, C. H. Sotak, Time-dependent diffusion of water in a biological model system., Proceedings of the National Academy of Sciences 91 (1994) 1229-1233. 
[14] S. Torquato, M. D. Rintoul, Effect of the interface on the properties of composite media, Phys. Rev. Lett. 75 (1995) 4067-.

[15] A. Szafer, J. Zhong, J. C. Gore, Theoretical model for water diffusion in tissues, Magn. Reson. Med. 33 (1995) 697-712.

[16] D. A. Yablonskiy, A. L. Sukstanskii, Theoretical models of the diffusion weighted MR signal, NMR Biomed. 23 (2010) 661-681.

[17] T. Niendorf, R. M. Dijkhuizen, D. G. Norris, M. van Lookeren Campagne, K. Nicolay, Biexponential diffusion attenuation in various states of brain tissue: Implications for diffusion-weighted imaging, Magn. Reson. Med. 36 (1996) 847-857.

[18] R. V. Mulkern, H. Gudbjartsson, C.-F. Westin, H. P. Zengingonul, W. Gartner, C. R. G. Guttmann, R. L. Robertson, W. Kyriakos, R. Schwartz, D. Holtzman, F. A. Jolesz, S. E. Maier, Multi-component apparent diffusion coefficients in human brain, NMR Biomed. 12 (1999) $51-62$.

[19] C. A. Clark, D. Le Bihan, Water diffusion compartmentation and anisotropy at high b values in the human brain, Magn. Reson. Med. 44 (2000) 852-859.

[20] S. E. Maier, P. Bogner, G. Bajzik, H. Mamata, Y. Mamata, I. Repa, F. A. Jolesz, R. V. Mulkern, Normal brain and brain tumor: Multicomponent apparent diffusion coefficient line scan imaging, Radiology 219 (2001) 842-849.

[21] S. Chabert, N. Molko, Y. Cointepas, P. Le Roux, D. Le Bihan, Diffusion tensor imaging of the human optic nerve using a non-CPMG fast spin echo sequence, J. Magn. Reson. Imaging 22 (2005) 307-310.

[22] J. H. Jensen, J. A. Helpern, A. Ramani, H. Lu, K. Kaczynski, Diffusional kurtosis imaging: The quantification of non-Gaussian water diffusion by means of magnetic resonance imaging, Magnetic Resonance in Medicine 53 (2005) 1432-1440.

[23] R. L. Magin, O. Abdullah, D. Baleanu, X. J. Zhou, Anomalous diffusion expressed through fractional order differential operators in the BlochTorrey equation, Journal of Magnetic Resonance 190 (2008) 255 - 270. 
[24] D. Yablonskiy, L. Bretthorst, J. Ackerman, Statistical model for diffusion attenuated MR signal, Magnetic Resonance in Medicine 50 (2003) 664-669.

[25] D. S. Novikov, V. G. Kiselev, Effective medium theory of a diffusionweighted signal, NMR in Biomedicine 23 (2010) 682-697.

[26] D. S. Novikov, E. Fieremans, J. H. Jensen, J. A. Helpern, Random walks with barriers, Nat Phys 7 (2011) 508-514.

[27] D. S. Novikov, J. H. Jensen, J. A. Helpern, E. Fieremans, Revealing mesoscopic structural universality with diffusion, Proceedings of the National Academy of Sciences (2014).

[28] P. N. Sen, P. J. Basser, A model for diffusion in white matter in the brain, Biophys J 89 (2005) 2927-2938.

[29] Y. Assaf, T. Blumenfeld-Katzir, Y. Yovel, P. J. Basser, Axcaliber: A method for measuring axon diameter distribution from diffusion MRI, Magn. Reson. Med. 59 (2008) 1347-1354.

[30] S. N. Jespersen, C. D. Kroenke, L. stergaard, J. J. Ackerman, D. A. Yablonskiy, Modeling dendrite density from magnetic resonance diffusion measurements, NeuroImage 34 (2007) 1473-1486.

[31] J. Karger, NMR self-diffusion studies in heterogeneous systems, Advances in Colloid and Interface Science 23 (1985) 129-148.

[32] J. Coatléven, H. Haddar, J.-R. Li, A new macroscopic model including membrane exchange for diffusion MRI, SIAM Journal of Applied Mathematics (2014).

[33] T. Arbogast, Gravitational forces in dual-porosity systems: I. Model derivation by homogenization, Transport in Porous Media 13 (1993) 179-203.

[34] T. Arbogast, Gravitational forces in dual-porosity systems: II. Computational validation of the homogenized model, Transport in Porous Media 13 (1993) 205-220. 
[35] A. Waldeck, P. W. Kuchel, A. J. Lennon, B. E. Chapman, NMR diffusion measurements to characterise membrane transport and solute binding, Progress in Nuclear Magnetic Resonance Spectroscopy 30 (1997) 39-68.

[36] G. J. Stanisz, G. A. Wright, R. M. Henkelman, A. Szafer, An analytical model of restricted diffusion in bovine optic nerve, Magn. Reson. Med. 37 (1997) 103-111.

[37] J. Pfeuffer, U. Flogel, W. Dreher, D. Leibfritz, Restricted diffusion and exchange of intracellular water: theoretical modelling and diffusion time dependence of 1H NMR measurements on perfused glial cells, NMR in Biomedicine 11 (1998) 19-31.

[38] J.-H. Lee, C. S. Springer, Effects of equilibrium exchange on diffusionweighted NMR signals: The diffusigraphic shutter-speed?, Magn. Reson. Med. 49 (2003) 450-458.

[39] C. Meier, W. Dreher, D. Leibfritz, Diffusion in compartmental systems. i. a comparison of an analytical model with simulations, Magnetic Resonance in Medicine 50 (2003) 500-509.

[40] Y. Roth, A. Ocherashvilli, D. Daniels, J. Ruizcabello, S. Maier, A. Orenstein, Y. Mardor, Quantification of water compartmentation in cell suspensions by diffusion-weighted and T2-weighted MRI, Magnetic Resonance Imaging 26 (2008) 88-102.

[41] I. Aslund, A. Nowacka, M. Nilsson, D. Topgaard, Filter-exchange PGSE NMR determination of cell membrane permeability, Journal of Magnetic Resonance 200 (2009) $291-295$.

[42] M. Nilsson, J. Latt, E. Nordh, R. Wirestam, F. Stahlberg, S. Brockstedt, On the effects of a varied diffusion time in vivo: is the diffusion in white matter restricted?, Magnetic Resonance Imaging 27 (2009) 176-187.

[43] E. Fieremans, D. S. Novikov, J. H. Jensen, J. A. Helpern, Monte Carlo study of a two-compartment exchange model of diffusion, NMR in Biomedicine 23 (2010) 711-724.

[44] H. Torrey, Bloch equations with diffusion terms, Physical Review Online Archive (Prola) 104 (1956) 563-565. 
[45] W. S. Price, A. V. Barzykin, K. Hayamizu, M. Tachiya, A model for diffusive transport through a spherical interface probed by pulsed-field gradient NMR, Biophysical Journal 74 (1998) 2259-2271.

[46] E. O. Stejskal, J. E. Tanner, Spin diffusion measurements: Spin echoes in the presence of a time-dependent field gradient, The Journal of Chemical Physics 42 (1965) 288-292.

[47] J. Xu, M. Does, J. Gore, Numerical study of water diffusion in biological tissues using an improved finite difference method, Physics in medicine and biology 52 (2007).

[48] J. Karger, H. Pfeifer, W. Heinik, Principles and application of selfdiffusion measurements by nuclear magnetic resonance, Advances in magnetic resonance 12 (1988) 1-89.

[49] A. Bensoussan, J.-L. Lions, G. Papanicolaou, Asymptotic analysis for periodic structures, volume 5 of Studies in Mathematics and its Applications, North-Holland Publishing Co., Amsterdam, 1978.

[50] R. Karlicek Jr., I. Lowe, A modified pulsed gradient technique for measuring diffusion in the presence of large background gradients, Journal of Magnetic Resonance (1969) 37 (1980) 75-91.

[51] D. V. Nguyen, J.-R. Li, D. Grebenkov, D. Le Bihan, A finite elements method to solve the Bloch-Torrey equation applied to diffusion magnetic resonance imaging, Journal of Computational Physics 263 (2014) 283302. 